\title{
Trigger factors in asthma and chronic obstructive pulmonary disease: a single-centre cross-sectional survey
}

\author{
Kay Choong $\underline{\text { See }}{ }^{1,2}$, FRCP, Jason $\underline{\text { Phua }}{ }^{1,2}$, FRCP, Tow Keang $\underline{\underline{L i m}^{1,2}}$, FRCP
}

\begin{abstract}
INTRODUCTION The presence of trigger factors may help to distinguish asthma from chronic obstructive pulmonary disease (COPD). Knowing and avoiding trigger factors for both asthma and COPD can facilitate the design of comprehensive management programmes that can aid disease control. This study aimed to describe the relative frequency and range of various trigger factors in asthma and COPD.

METHODS We conducted a telephone-based survey involving asthma and COPD patients on follow-up at a university hospital in Singapore.

RESULTS A total of 779 asthma patients and 129 COPD patients participated in this study. Among these patients, $93.8 \%$ of those with asthma and $42.6 \%$ of those with COPD had trigger factors $(p<0.001)$. The median number of trigger factors was greater among asthma patients than among those with COPD ( 3 vs. $0, p<0.001$ ). Trigger factors found to be significantly more prevalent among asthma patients compared to those with COPD include tobacco smoke, alcohol, upper respiratory tract infections, incense smoke, perfume, laughter, a dusty environment, air-conditioning, heavy rain, heavy traffic fumes, citrus fruits, gastro-oesophageal reflux, household pets, flowers/pollen, medications and psychological triggers. Trigger factors that were not previously described, such as bathing, fatigue, insufficient sleep, crowded places and overeating, were also reported.

CONCLUSION Trigger factors, although found in both groups of patients, were more common among asthma patients. Knowledge of these trigger factors may be useful in distinguishing between the two diseases and optimising disease management.
\end{abstract}

Keywords: asthma, chronic disease, chronic obstructive pulmonary disease, disease management, precipitating factors

\section{INTRODUCTION}

The latest report from the Global Initiative for Asthma (GINA) suggested that trigger factors could be used to distinguish asthma from chronic obstructive pulmonary disease (COPD). ${ }^{(1)}$ While this is a reasonable recommendation, there is a lack of published empirical data to show that the presence or frequency of trigger factors differs between these two common respiratory conditions. There is also little evidence to show that trigger factors may be useful in differentiating asthma from COPD. . $^{(2,3)}$

Avoidance of trigger factors that exacerbate asthma and COPD is an important part of disease control, particularly if the disease is refractory to usual treatment. ${ }^{(4-7)}$ Even if the trigger factors are not completely avoidable, attempts to reduce their effects may still be possible; for example, temporary escalation of therapy can be performed during periods of exposure. ${ }^{(8)}$ Medical advice would then depend on knowledge of the types and range of probable trigger factors for both asthma and COPD. However, most prior studies on trigger factors focused on one or a few trigger factors, and generally involved small sample sizes; they were also mainly performed in Western populations and lack information on COPD. ${ }^{(9,10)}$ Therefore, the present study aimed to comprehensively describe the prevalence and range of trigger factors for both asthma and COPD, and compare their relative frequency.

\section{METHODS}

All patients with asthma or COPD who were on active follow-up with respiratory physicians in our 1,000-bed hospital in Singapore, as of 30 April 2009, were enrolled in the present study. The diagnosis of asthma or COPD was made based on the physician's assessment, which was done in accordance with standard guidelines. ${ }^{(11,12)}$ Spirometry data, which was obtained based on the American Thoracic Society/European Respiratory Society Task Force guidelines, ${ }^{(13)}$ was extracted from the pulmonary function laboratory's computerised records and referenced using local norms. ${ }^{(14)}$ The present study was approved by the National Healthcare Group Domain Specific Review Board (reference no. B/09/040) and verbal informed consent was obtained from all participants.

Research nurses administered a telephone-based questionnaire to patients to enquire if particular trigger factors worsened their disease condition, i.e. led to increased breathlessness, wheezing or coughing (Box 1). A wide range of specific trigger factors (including previously investigated factors and others that were derived from our clinical experience) were covered in the questionnaire. These trigger factors included tobacco smoke, alcohol, ${ }^{(15)}$ upper respiratory tract infections, ${ }^{(16)}$ incense smoke, ${ }^{(17)}$ perfume, chemical exposure at work, laughter, ${ }^{(18)}$ a dusty environment, air-conditioning, hot weather, cold weather, heavy rain, ${ }^{(19)}$ heavy traffic fumes, citrus fruits, other foods, ${ }^{(20)}$

${ }^{1}$ Division of Respiratory and Critical Care Medicine, University Medicine Cluster, National University Health System, ${ }^{2}$ Department of Medicine, Yong Loo Lin School of Medicine, National University of Singapore, Singapore

Correspondence: Dr See Kay Choong, Consultant, Division of Respiratory and Critical Care Medicine, University Medicine Cluster, National University Health System, 1E Kent Ridge Road, NUHS Tower Block Level 10, Singapore 119228. kay_choong_see@nuhs.edu.sg 


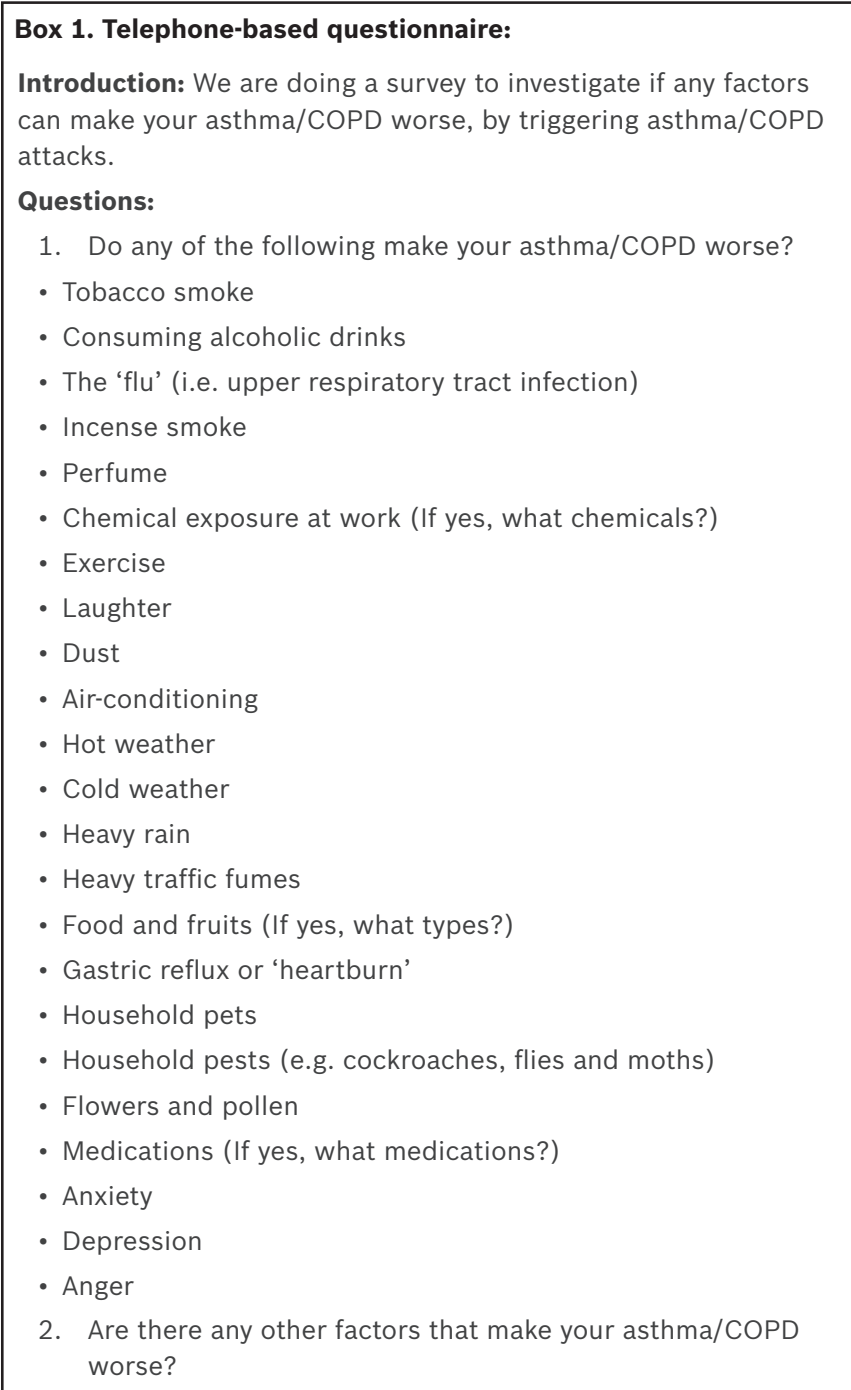

gastro-oesophageal reflux symptoms (e.g. heartburn), household pets, household pests, ${ }^{(21)}$ flowers (i.e. pollen), ${ }^{(22)}$ medications ${ }^{(23)}$ and psychological triggers (e.g. anxiety, depression and anger). ${ }^{(24)}$ The questionnaire included an open-ended question that asked patients about any other trigger factors known to aggravate their condition.

Statistical analysis was performed using SPSS Statistics version 17.0 for Windows (SPSS Inc, Chicago, IL, USA). Results were presented as mean \pm standard deviation, or median (interquartile range [IQR]). Fisher's exact test was used for nominal data, Mann-Whitney $U$ test was used for ordinal data and Student's $t$-test was used for continuous data (after verifying normality and equality of variances). Baseline characteristics and the frequency of various trigger factors among the patients with asthma and those with COPD were compared. We described summary data for miscellaneous trigger factors that were not specifically addressed in the questionnaire. A p-value $<0.05$ indicated statistical significance and all tests performed were two-tailed.

\section{RESULTS}

A total of 1,289 asthma patients and 192 COPD patients were eligible for inclusion in the study. Among these patients, $779(60.4 \%)$ asthma patients and 129 (67.2\%) COPD patients
Table I. Characteristics of the patients who participated in the survey $(n=908)$.

\begin{tabular}{lccc}
\hline Characteristic & \multicolumn{2}{c}{ Mean \pm standard deviation } & \multirow{2}{*}{ p-value } \\
\cline { 2 - 3 } & $\begin{array}{c}\text { Asthma } \\
(\mathbf{n}=\mathbf{7 7 9})\end{array}$ & $\begin{array}{c}\text { COPD } \\
(\mathbf{n}=\mathbf{1 2 9})\end{array}$ & \\
\hline Age (yr) & $47.9 \pm 18.1$ & $74.0 \pm 8.5$ & $<0.001$ \\
Male gender* & $375(48.1)$ & $117(90.7)$ & $<0.001$ \\
Currently smoking* & $94(12.1)$ & $97(75.2)$ & $<0.001$ \\
Spirometry data & & & \\
(\% predicted) & & & \\
Pre-BD FEV1 & $69.5 \pm 26.8$ & $46.5 \pm 17.5$ & $<0.001$ \\
Post-BD FEV1 & $74.7 \pm 26.7$ & $48.9 \pm 17.8$ & $<0.001$ \\
Pre-BD FVC & $73.9 \pm 16.3$ & $59.1 \pm 15.6$ & $<0.001$ \\
Post-BD FVC & $77.4 \pm 16.0$ & $63.5 \pm 15.6$ & $<0.001$ \\
GOLD clinical stage* & & & \\
I & NA & $5(3.9)$ & NA \\
II & NA & $46(35.7)$ & NA \\
III & NA & $62(48.1)$ & NA \\
IV & NA & $16(12.4)$ & NA \\
\hline
\end{tabular}

*Data presented as no. (\%). BD: bronchodilator; COPD: chronic obstructive pulmonary disease; FEV1: forced expiratory volume in the 1st second; FVC: forced vital capacity; GOLD: Global Initiative for Chronic Obstructive Lung Disease; NA: not applicable

agreed to participate in the telephone survey. The overall participation rate was $61.3 \%$.

The overall ethnic distribution of the patients was $52.3 \%$ Chinese, $34.3 \%$ Malay, $11.5 \%$ Indian and $2.0 \%$ other ethnicities. Among the survey participants, spirometry data was found for $684(87.8 \%)$ asthma and $122(94.6 \%)$ COPD patients. Patients with COPD were more likely to be of older age, male and currently smoking tobacco, as compared to those with asthma (Table I). As expected, COPD patients also had significantly poorer lung function than those with asthma; they also predominantly had moderately severe disease (i.e. Stage III), as defined by the Global Initiative for Chronic Obstructive Lung Disease. ${ }^{(12)}$

We found that more than twice the number of asthma patients had at least one trigger factor as compared to COPD patients $(93.8 \%$ vs. $42.6 \%, p<0.001)$. In addition, more asthma patients had multiple trigger factors. The median number of trigger factors was also greater among asthma patients than among those with COPD (3 [IQR 2-4] vs. 0 [IQR 0-1], p < 0.001; Table II). All the trigger factors surveyed were significantly more prevalent among asthma patients than COPD patients, except for chemical exposure at work, hot weather and household pests (these three factors were not significantly different between the two groups). In this survey, we reported several trigger factors that, to the best of our knowledge, were not previously described, including bathing, fatigue, insufficient sleep, crowded places and overeating.

\section{DISCUSSION}

In this study, we showed that there is a wide range of trigger factors for asthma and COPD, and identified novel trigger factors such as bathing, fatigue, insufficient sleep, crowded places and overeating. A substantial proportion of the participants had trigger factors that exacerbated their symptoms ( $93.8 \%$ of asthma patients 
Table II. Trigger factors reported by the asthma or chronic obstructive pulmonary disease (COPD) patients $(n=908)$.

\begin{tabular}{|c|c|c|c|}
\hline \multirow[t]{2}{*}{ Variable } & \multicolumn{2}{|c|}{$\%$} & \multirow[t]{2}{*}{ p-value } \\
\hline & $\begin{array}{l}\text { Asthma } \\
(n=779)\end{array}$ & $\begin{array}{c}\text { COPD } \\
(n=129)\end{array}$ & \\
\hline No. of trigger factors* & $3(2-4)$ & $0(0-1)$ & $<0.001$ \\
\hline$\geq 1$ & 93.8 & 42.6 & $<0.001$ \\
\hline$\geq 2$ & 81.4 & 17.8 & $<0.001$ \\
\hline$\geq 3$ & 60.9 & 11.6 & $<0.001$ \\
\hline \multicolumn{4}{|l|}{ Trigger factor } \\
\hline Dust & 63.2 & 16.3 & $<0.001$ \\
\hline $\begin{array}{l}\text { Heavy rain } \\
\text { (cold and wet weather) }\end{array}$ & 32.5 & 17.1 & $<0.001$ \\
\hline $\begin{array}{l}\text { Upper respiratory tract } \\
\text { infection }\end{array}$ & 29.5 & 9.3 & $<0.001$ \\
\hline Anxiety/anger/depression & 23.1 & 7.0 & $<0.001$ \\
\hline Heavy traffic fumes & 20.2 & 0.8 & $<0.001$ \\
\hline Citrus fruits & 19.9 & 1.6 & $<0.001$ \\
\hline Air-conditioning & 19.1 & 3.1 & $<0.001$ \\
\hline Tobacco smoke & 15.7 & 2.3 & $<0.001$ \\
\hline Hot weather & 14.9 & 12.4 & 0.503 \\
\hline Incense smoke & 14.8 & 1.6 & $<0.001$ \\
\hline Laughter & 11.2 & 1.6 & $<0.001$ \\
\hline Household pets & 10.0 & 0 & $<0.001$ \\
\hline Perfume & 9.5 & 0.8 & $<0.001$ \\
\hline Flowers/pollen & 6.3 & 0 & 0.001 \\
\hline Alcohol & 5.1 & 0.8 & 0.021 \\
\hline $\begin{array}{l}\text { Gastro-oesophageal } \\
\text { reflux (i.e. heartburn) }\end{array}$ & 5.1 & 0.8 & 0.021 \\
\hline Medication & 3.3 & 0 & 0.04 \\
\hline Chemical exposure at work & 3.2 & 2.3 & 0.786 \\
\hline Household pests ${ }^{\dagger}$ & 2.1 & 0 & 0.148 \\
\hline Others & 14.4 & 2.3 & $<0.001$ \\
\hline
\end{tabular}

*Data presented as median (interquartile range). ${ }^{\dagger}$ Includes cockroaches, flies and moths. \#Includes bathing, fatigue, insufficient sleep, crowded places and overeating.

and $42.6 \%$ of COPD patients). Asthma patients were found to have more trigger factors than those with COPD (median 3 vs. 0),

More than $90 \%$ of the asthma patients in our study reported at least one trigger factor. Qualitatively, some of the trigger factors examined in the present study, such as laughter and anxiety, have been proposed to be markers of suboptimal asthma control and near-fatal asthma. ${ }^{(25,26)}$ Quantitatively, an increased number of trigger factors has been linked to poorer asthma control. ${ }^{(27,28)}$ As asthma triggers adversely affect a patient's well-being and primary care utilisation, the inability to identify trigger factors is a risk factor for sudden-onset asthmatic exacerbations and can result in visits to the emergency department. ${ }^{(29,30)}$ In contrast to asthma, little is known about the trigger factors of COPD, particularly their role in the control of COPD symptoms and exacerbation.

The immunological, inflammatory, neural and other postulated mechanisms by which exogenous and endogenous trigger factors aggravate asthma and COPD are largely uncertain. ${ }^{(31)}$ In the present study, the frequency of trigger factors was higher among asthma patients than among COPD patients.
This is likely associated with the underlying pathophysiology of the two diseases. For instance, atopy has been shown (in research using clinical questionnaires) to be associated with a diagnosis of asthma. ${ }^{(32)}$ However, there is generally no recognised relationship between atopy and COPD. Nonetheless, non-atopic mechanisms, such as the direct irritation of diseased airways, could occur and may account for the presence of trigger factors in COPD.

One strength of the present study is its relatively large sample size, making it one of the largest studies to examine trigger factors of asthma. We were therefore able to explore a larger range of potential contributors. This is also one of the few studies that investigated the trigger factors of COPD. Furthermore, while several trigger factors found in our tropical context are also found in temperate conditions, the local climate and culture allowed us to explore the effects of triggers such as heavy rain and exposure to incense smoke.

The present study also had several limitations. Firstly, the use of a questionnaire for data collection subjected the data to recall bias. Nevertheless, this is similar to actual clinical practice where history-taking is the primary method for identifying trigger factors. Secondly, the response rate was moderate at $61.3 \%$. However, as the resulting sample size was still fairly large, the findings were likely to be representative of our patient cohort. Thirdly, we used the cohort of asthma and COPD patients from our hospital's follow-up database, which could impact the applicability of the results to patients on follow-up with primary care physicians. Nonetheless, the patients who would benefit the most from additional information on disease management are most likely to be those who require tertiary care. Fourthly, we did not collect detailed clinical and laboratory data on the baseline severity and control of asthma, and skin prick testing was not routinely performed for our patients. These factors, however, would not have affected the results or conclusions of the present study. Finally, previously validated questionnaires (e.g. for trigger factors, anxiety and depression) were not used in this study, as we wanted to minimise the survey burden and maximise participation. ${ }^{(33,34)}$

The clinical implications of the present study are threefold. Firstly, we empirically validated the recommendation by GINA to use trigger factors for distinguishing asthma from COPD. Based on the findings of the present study, it is also possible that COPD patients with multiple trigger factors may have asthma-COPD overlap syndrome, which has been associated with increased illness severity and healthcare utilisation. ${ }^{(7,35,36)}$ In other words, knowledge of trigger factors may help to identify this important clinical phenotype.

Secondly, our findings suggest that the importance of trigger factors in COPD is under-recognised. We highlight that although trigger factors are more common in asthma patients, ${ }^{(37)}$ they also occur frequently in COPD patients. In the present study, nearly half of the COPD patients had at least one trigger factor. Among COPD patients (as well as asthma patients), non-infectious triggers such as dust, hot weather and heavy rain were more frequently reported than infectious trigger factors such as upper respiratory 
tract infections; this is a fact that may not be widely appreciated. Specifically, the proportions of asthma patients with infectious triggers, pollutant-related triggers (e.g. tobacco smoke, heavy traffic fumes and incense smoke) and other non-infectious triggers (e.g. stress) were $29.5 \%, 33.5 \%$ and $90.6 \%$, respectively. The corresponding proportions for COPD patients were 9.3\%, 4.7\% and $38.8 \%$, respectively. Hence, trigger factor control can be valuable in the management of both COPD and asthma.

Thirdly, this study provided information on a wide range of trigger factors. This information can be used to facilitate comprehensive trigger factor management, either by avoidance or desensitisation. Prior research has shown that avoidance measures directed narrowly toward single allergens were not effective. ${ }^{(38)}$ On the other hand, a structured system of avoidance against multiple allergens has been shown to result in improved symptoms and spirometry-derived indices of lung function among patients with atopic asthma. ${ }^{(37,39)}$ It is our hope that the findings of the present study will help enhance management plans for asthma and COPD, as well as improve the advice given to asthma and COPD patients. For example, patients could be presented with the whole range of trigger factors explored in this study so that they can quickly identify the problematic ones. While we recognise that some environmental and climatic trigger factors are difficult to avoid (e.g. those that impair the patient's quality of life), we believe that most of the factors (e.g. tobacco smoke, perfume, certain types of foods and gastro-oesophageal reflux) can be readily mitigated. Furthermore, we noted that a substantial percentage of the patients in the present study were adversely affected by emotional changes (e.g. anxiety, anger or depression). This group of patients could potentially benefit from adjunctive psychological treatment strategies.

The present study helps to provide a fair idea of the range and proportions of trigger factors in asthma and COPD patients. The next step could be correlating these trigger factors to disease control, symptoms, exacerbation and healthcare utilisation. Although this was done for asthma patients in prior studies, ${ }^{(40,41)}$ there is a need for validation in the Asian setting. Clinically important trigger factors could be used to design management programmes that incorporate mitigation of exposure to aggravating substances. Similar questionnaire surveys could also be conducted in other settings to search for more unusual trigger factors. Although we were not able to offer a definite biological explanation for these novel trigger factors, the identification of such factors could still be useful in improving the management of asthma and COPD. For instance, if fatigue is found to trigger asthma or COPD, the relevant patients could be advised to ensure that they get sufficient rest or sleep.

In conclusion, a broad range of trigger factors exists for both asthma and COPD, although asthma patients tend to have a greater number of trigger factors. Knowledge of these trigger factors may help in the differential diagnosis of asthma and COPD, and mitigation of these trigger factors may improve disease management.

\section{ACKNOWLEDGEMENTS}

The authors sincerely thank the following collaborators for contributing to the data collection: the research nurses from the
School of Health Sciences, Ngee Ann Polytechnic, Singapore; Ms Dong-Xia Shi, Pulmonary Function Laboratory, National University Hospital, Singapore; and Ms Wang-Jee Ngerng, Division of Respiratory and Critical Care Medicine, National University Hospital, Singapore.

\section{REFERENCES}

1. Global Initiative for Asthma. Global Strategy for Asthma Management and Prevention, Global Initiative for Asthma 2014. Available at: http://www. ginasthma.org. Accessed November 1, 2014.

2. Thiadens HA, de Bock GH, Dekker FW, et al. Identifying asthma and chronic obstructive pulmonary disease in patients with persistent cough presenting to general practitioners: descriptive study. BMJ 1998; 316:1286-90.

3. Tinkelman DG, Price DB, Nordyke RJ, et al. Symptom-based questionnaire for differentiating COPD and asthma. Respiration 2006; 73:296-305.

4. Hunt LW. How to manage difficult asthma cases. An action plan for physicians and patients. Postgrad Med 2001; 109:61-8.

5. Bobb C, Ritz T, Rowlands G, Griffiths C. Effects of allergen and trigger factor avoidance advice in primary care on asthma control: a randomizedcontrolled trial. Clin Exp Allergy 2010; 40:143-52.

6. British Thoracic Society; Scottish Intercollegiate Guidelines Network. British guideline on the management of asthma. Thorax 2014; 69 Suppl 1:1-192.

7. Reddy AP, Gupta MR. Management of asthma: the current US and European guidelines. Adv Exp Med Biol 2014; 795:81-103.

8. McAlpine LG, Thomson NC. Prophylaxis of exercise-induced asthma with inhaled formoterol, a long-acting beta 2-adrenergic agonist. Respir Med 1990; 84:293-5.

9. Tarlo SM, Broder I, Corey P, et al. A case-control study of the role of cold symptoms and other historical triggering factors in asthma exacerbations. Can Respir J 2000; 7:42-8.

10. Weiss ST, Horner A, Shapiro G, Sternberg AL; Childhood Asthma Management Program (CAMP) Research Group. The prevalence of environmental exposure to perceived asthma triggers in children with mild-to-moderate asthma: data from the Childhood Asthma Management Program (CAMP). J Allergy Clin Immunol 2001; 107:634-40.

11. Bateman ED, Hurd SS, Barnes PJ, et al. Global strategy for asthma management and prevention: GINA executive summary. Eur Respir J 2008; 31:143-78.

12. Rabe KF, Hurd S, Anzueto A, et al; Global Initiative for Chronic Obstructive Lung Disease. Global strategy for the diagnosis, management, and prevention of chronic obstructive pulmonary disease: GOLD executive summary. Am J Respir Crit Care Med 2007; 176:532-55.

13. Miller MR, Hankinson J, Brusasco V, et al; ATS/ERS Task Force. Standardisation of spirometry. Eur Respir J 2005; 26:319-38.

14. da Costa JL. Pulmonary function studies in healthy Chinese adults in Singapore. Am Rev Respir Dis 1971; 104:128-31

15. Vally $\mathrm{H}$, de Klerk N, Thompson PJ. Alcoholic drinks: important triggers for asthma. J Allergy Clin Immunol 2000; 105:462-7.

16. Wedzicha JA, Donaldson GC. Exacerbations of chronic obstructive pulmonary disease. Respir Care 2003; 48:1204-13; discussion 1213-5.

17. Al-Rawas OA, Al-Maniri AA, Al-Riyami BM. Home exposure to Arabian incense (bakhour) and asthma symptoms in children: a community survey in two regions in Oman. BMC Pulm Med 2009; 9:23.

18. Liangas G, Yates DH, Wu D, Henry RL, Thomas PS. Laughter-associated asthma. J Asthma 2004; 41:217-21.

19. Khot A, Burn R, Evans N, Lenney W, Storr J. Biometeorological triggers in childhood asthma. Clin Allergy 1988; 18:351-8.

20. Nékám KL. Nutritional triggers in asthma. Acta Microbiol Immunol Hung 1998; 45:113-7.

21. Arif AA, Delclos GL, Lee ES, Tortolero SR, Whitehead LW. Prevalence and risk factors of asthma and wheezing among US adults: an analysis of the NHANES III data. Eur Respir J 2003; 21:827-33.

22. Brown CW, Hawkins L. Allergy prevalence and causal factors in the domestic environment: results of a random population survey in the United Kingdom. Ann Allergy Asthma Immunol 1999; 83:240-4.

23. Covar RA, Macomber BA, Szefler SJ. Medications as asthma triggers. Immunol Allergy Clin North Am 2005; 25:169-90.

24. Ritz T, Kullowatz A, Bobb C, et al. Psychological triggers and hyperventilation symptoms in asthma. Ann Allergy Asthma Immunol 2008; 100:426-32.

25. Mitchell I, Tough SC, Semple LK, Green FH, Hessel PA. Near-fatal asthma: 
a population-based study of risk factors. Chest 2002; 121:1407-13.

26. Liangas G, Morton JR, Henry RL. Mirth-triggered asthma: is laughter really the best medicine? Pediatr Pulmonol 2003; 36:107-12.

27. de Vries MP, van den Bemt $L$, Lince $S$, et al. Factors associated with asthma control. J Asthma 2005; 42:659-65.

28. Emerman CL, Woodruff PG, Cydulka RK, et al. Prospective multicenter study of relapse following treatment for acute asthma among adults presenting to the emergency department. MARC investigators. Multicenter Asthma Research Collaboration. Chest 1999; 115:919-27.

29. Ritz T, Kullowatz A, Kanniess F, Dahme B, Magnussen H. Perceived triggers of asthma: evaluation of a German version of the Asthma Trigger Inventory. Respir Med 2008; 102:390-8.

30. Woodruff PG, Emond SD, Singh AK, Camargo CA Jr. Sudden-onset severe acute asthma: clinical features and response to therapy. Acad Emerg Med 1998; 5:695-701.

31. Murray CS, Simpson A, Custovic A. Allergens, viruses, and asthma exacerbations. Proc Am Thorac Soc 2004; 1:99-104.

32. Beeh KM, Kornmann O, Beier J, Ksoll M, Buhl R. Clinical application of a simple questionnaire for the differentiation of asthma and chronic obstructive pulmonary disease. Respir Med 2004; 98:591-7.

33. Liu S, Wu R, Li L, et al. The prevalence of anxiety and depression in Chinese asthma patients. PLoS One 2014; 9:e103014.
34. Ritz T, Steptoe A, Bobb C, Harris AH, Edwards M. The asthma trigger inventory: validation of a questionnaire for perceived triggers of asthma. Psychosom Med 2006; 68:956-65.

35. Braman SS. The chronic obstructive pulmonary disease-asthma overlap syndrome. Allergy Asthma Proc 2015; 36:11-8.

36. Boulet LP, FitzGerald JM, Reddel HK. The revised 2014 GINA strategy report: opportunities for change. Curr Opin Pulm Med 2015; 21:1-7.

37. Bobb C, Ritz T. Do asthma patients in general practice profit from a structured allergy evaluation and skin testing? A pilot study. Respir Med 2003; 97:1180-7.

38. Woodcock A, Forster L, Matthews E, et al; Medical Research Council General Practice Research Framework. Control of exposure to mite allergen and allergen-impermeable bed covers for adults with asthma. N Engl J Med $2003 ; 349: 225-36$

39. Morgan WJ, Crain EF, Gruchalla RS, et al; Inner-City Asthma Study Group. Results of a home-based environmental intervention among urban children with asthma. N Engl J Med 2004; 351:1068-80.

40. Price D, Dale P, Elder E, Chapman KR. Types, frequency and impact of asthma triggers on patients' lives: a quantitative study in five European countries. J Asthma 2014; 51:127-35.

41. Murray CS, Poletti G, Kebadze T, et al. Study of modifiable risk factors for asthma exacerbations: virus infection and allergen exposure increase the risk of asthma hospital admissions in children. Thorax 2006; 61:376-82. 